\title{
COMPÓSITOS COBRE-GRAFENO PRODUZIDOS POR METALURGIA DO PÓ COM COMPACTAÇÃO A FRIO *
}

\author{
Géssica Seara Faria ${ }^{1}$ \\ Andreza Menezes Lima² \\ Wagner Anacleto Pinheiro ${ }^{3}$ \\ Alexandre Antunes Ribeiro ${ }^{4}$ \\ Luiz Paulo Mendonça Brandão ${ }^{5}$
}

\section{Resumo}

Foram fabricados compósitos de matriz de cobre, reforçados com grafeno a partir da mistura de uma dispersão de óxido de grafeno com pó de cobre eletrolítico. A dispersão foi produzida pelo método de Hummers modificado e caracterizada por espectroscopia Raman e difração de raios X. Os compósitos foram fabricados pelo processo de metalurgia do pó convencional. Os pós de compósitos foram caracterizados por microscopia eletrônica de varredura, os corpos de prova consolidados foram avaliados quanto a densificação a verde e do corpo sinterizado, e os corpos sinterizados foram submetidos a ensaio de compressão a fim de verificar a resistência dos compósitos.

Palavras-chave: Óxido de grafeno; Compósitos de matriz metálica; Metalurgia do pó.

\section{COPPER-GRAPHENE COMPOSITES PRODUCED BY COLD DIE COMPACTATION OF POWDER METALLURGY}

\begin{abstract}
Copper matrix composites reinforced with graphene were produced from mixing graphene oxide aqueous dispersion with electrolytic copper powders. The dispersion was produced by the modified Hummers method and characterized by Raman spectroscopy and X-ray diffraction. The composites were produced using powder metallurgy process with cold die compaction. The composite powders were characterized by scanning electron microscopy, the bulk composites were evaluated as its green and sintered densification and sintered bulk composites were subjected to a compression test in order to verify the resistance.
\end{abstract}

Keywords: Graphene oxide; Metal matrix composites; Powder Metallurgy.

1 Engenheira Metalúrgica, mestranda em Ciências dos Materiais, SE/4 - Seção de ensino de Engenharia Mecânica e de Materiais, Instituto Militar de Engenharia, Rio de Janeiro, RJ, Brasil.

2 Engenheira Mecânica, doutoranda em Ciências dos Materiais, SE/4 - Seção de ensino de Engenharia Mecânica e de Materiais, Instituto Militar de Engenharia, Rio de Janeiro, RJ, Brasil.

3 Engenheiro Metalúrgico, doutor em Ciências dos Materiais, professor/coordenador, SE/4 - Seção de ensino de Engenharia Mecânica e de Materiais, Instituto Militar de Engenharia, Rio de Janeiro, RJ, Brasil.

4 Engenheiro Químico, doutor em Engenharia Química, pesquisador, Divisão de Processamento e Caracterização de Materiais/Laboratório de Tecnologia de Pós, Instituto Nacional de Tecnologia, Rio de Janeiro, RJ, Brasil.

5 Engenheiro Metalúrgico, doutor em Engenharia Metalúrgica e de Materiais, professor, SE/4 Seção de ensino de Engenharia Mecânica e de Materiais, Instituto Militar de Engenharia, Rio de Janeiro, RJ, Brasil. 


\section{INTRODUÇÃO}

A indústria da metalurgia do pó é amplamente utilizada para fabricação de uma grande variedade de produtos. A técnica apresenta inúmeras vantagens como a produção e conformação de metais muito difíceis de serem obtidos por processos convencionais, obtenção de características especiais como porosidade controlada, associação de metais com materiais não metálicos e possibilidade de controle rigoroso do produto acabado [1].

O grafeno, descoberto em 2004, tem sido largamente estudado nos últimos anos, por se tratar de um material com propriedades excepcionais como alta resistência mecânica, condutividade elétrica e térmica, proporcionada pela sua estrutura em camadas hexagonais 2D [2,3,4]. Uma das inúmeras possibilidades de utilização deste material é como reforço para materiais com matriz metálica [5]. Desta forma, a técnica de metalurgia do pó se mostra adequada para fabricação e avaliação de compósitos formados por materiais metálicos reforçados com grafeno.

Este trabalho tem como objetivo avaliar compósitos com matriz de cobre, reforçados com grafeno na forma de óxido de grafeno (graphene oxide - GO), fabricados por metalurgia do pó, de modo que ocorra redução térmica durante a sinterização, formando óxido de grafeno reduzido (reduced graphene oxide - rGO) e restaurando assim a forma original do grafeno.

\section{MATERIAIS E MÉTODOS}

\subsection{Preparação do GO}

A dispersão aquosa de $\mathrm{GO}$ foi preparada pelo método de Hummers modificado [6] a partir do grafite Graflake 99550 fornecido pela empresa Nacional de Grafite. O processo consistiu em adições de ácido sulfúrico e nitrato de sódio ao grafite, para promover a intercalação. Em seguida foram realizadas adições de permanganato de potássio com intervalos de 10 minutos e ao final das adições a mistura foi mantida sobre agitação durante 7 dias, o que resultou na oxidação do grafite. A esfoliação inicial das camadas da mistura foi realizada por meio da adição de uma solução diluída de ácido sulfúrico e peróxido de hidrogênio. Por fim foram realizadas repetidas etapas de lavagem e centrifugação, para concluir a esfoliação e eliminar reagentes em excesso da mistura.

\subsection{Fabricação dos pós de compósitos $\mathrm{Cu} / \mathrm{GO}$}

A dispersão aquosa de GO foi diluída em $350 \mathrm{ml}$ de água, utilizando 266 e $480 \mathrm{ml}$ de GO (concentração inicial de 2,46 e 2,72 $\mathrm{mg} / \mathrm{ml}$ ) e adicionados $162 \mathrm{~g}$ de cobre para produzir compósitos com 0,4 e $0,8 \%$ em peso de GO, respectivamente. A mistura permaneceu em agitação mecânica durante 1 hora e foi, posteriormente, filtrada a vácuo. Os pós de compósitos cobre-óxido de grafeno (Cu-GO), foram obtidos após secagem a vácuo durante $48 \mathrm{~h}$.

\subsection{Consolidação dos corpos de prova dos compósitos $\mathrm{Cu} / \mathrm{GO}$}

Os pós de compósitos foram moldados por meio de uma matriz cilíndrica com 12 $\mathrm{mm}$ de diâmetro e pelo processo de prensagem uniaxial a frio, com pressão de 87 
MPa. A massa do pó de cobre e pós de compósitos adicionada foi de $12 \mathrm{~g}$, com o objetivo de obter um corpo de prova com relação diâmetro/altura (H:D) de 1:1.

A sinterização foi realizada em alto vácuo (10-6 Torr), de maneira a promover a redução do $\mathrm{GO}$, temperatura $820^{\circ} \mathrm{C}$ com patamar de 2 horas e taxa de aquecimento de $10^{\circ} \mathrm{C} / \mathrm{min}$.

\subsection{Caracterizações}

Para confirmar a obtenção do GO foi realizado teste de espectroscopia Raman utilizando o espectrômetro confocal NT-MDT NTERGRA, com comprimento de onda do laser de $473 \mathrm{~nm}$, faixa de varredura entre 198 e $3336 \mathrm{~cm}^{-1}$ e tempo de radiação de $100 \mathrm{~s}$.

A técnica de difração de raios $X(D R X)$ foi empregada utilizando o equipamento X'Pert Pro MPD da PANalytical com o tubo de radiação $\mathrm{Cu} K a(\lambda=1,54 \AA)$, configurado em foco linha, empregando a geometria de Bragg-Bretano. Os parâmetros operacionais utilizados foram tensão e corrente de $45 \mathrm{kV}$ e $40 \mathrm{~mA}$, respectivamente, com intervalo de varredura em $2 \theta$ de 5 a $40^{\circ}$, passo de $0,05^{\circ}$ e tempo de 2,5 s por passo para as amostras de GO.

Os pós de compósitos foram analisados por microscopia eletrônica de varredura (MEV) em um microscópio Inspect S50 da marca FEI, com ampliação de 2500 x.

A densificação dos corpos de prova a verde e sinterizados foi obtida pelo método geométrico que relaciona a massa e volume do material pelas equações 1 e 2 , em comparação a densidade teórica do cobre de $8,94 \mathrm{~g} / \mathrm{cm}^{3}$.:

$$
\begin{array}{r}
\rho_{\text {amostra }}=\frac{m}{V} \\
\operatorname{DR}(\%)=\left(\frac{\rho_{\text {amostra }}}{\rho_{\text {teórica }}}\right) \times 100
\end{array}
$$

onde,

$\boldsymbol{\rho}_{\text {amostra }}=$ densidade calculada $\left(\mathrm{g} / \mathrm{cm}^{3}\right)$;

$\boldsymbol{m}=$ massa da amostra $(\mathrm{g})$;

$\boldsymbol{V}=$ volume da amostra $\left(\mathrm{cm}^{3}\right)$;

$\boldsymbol{D} \boldsymbol{R}=$ densidade relativa $(\%)$;

$\boldsymbol{\rho}_{\text {teórica }}=$ densidade do cobre $\left(8,94 \mathrm{~g} / \mathrm{cm}^{3}\right)$.

Os corpos de prova (CP's) obtidos foram submetidos a testes de compressão. A velocidade do ensaio foi variável de modo a manter a taxa de $0,000212 \mathrm{~s}^{-1}$ para todos os CP's. A resistência mecânica à compressão do material obtido foi analisada a $40 \%$ de deformação.

\section{RESULTADOS E DISCUSSÃO}

A Figura 1 mostra os espectros Raman obtidos para a dispersão de GO produzida. Foram identificadas as bandas D e G com deslocamento Raman de 1367 e $1608 \mathrm{~cm}^{-}$ 1 , respectivamente. $A$ banda $D$ é relacionada aos grupos funcionais de oxigênio ligados as camadas de carbono, enquanto que a banda $\mathrm{G}$ é devido a hibridização $\mathrm{sp}^{2}$, relacionada aos materiais carbonosos [7]. A relação de intensidade entre as bandas $D$ e $G\left(I_{D} / I_{G}\right)$ é uma maneira de medir a quantidade de defeitos presente no $\mathrm{GO}$, considerando que quanto maior o valor de $\mathrm{I}_{\mathrm{D}} / \mathrm{I}_{\mathrm{G}}$ maior será a densidade de defeitos presentes na estrutura. Neste estudo a razão encontrada foi de 1,116, o que indica uma grande quantidade de defeitos existentes no GO, gerados na etapa de oxidação. 


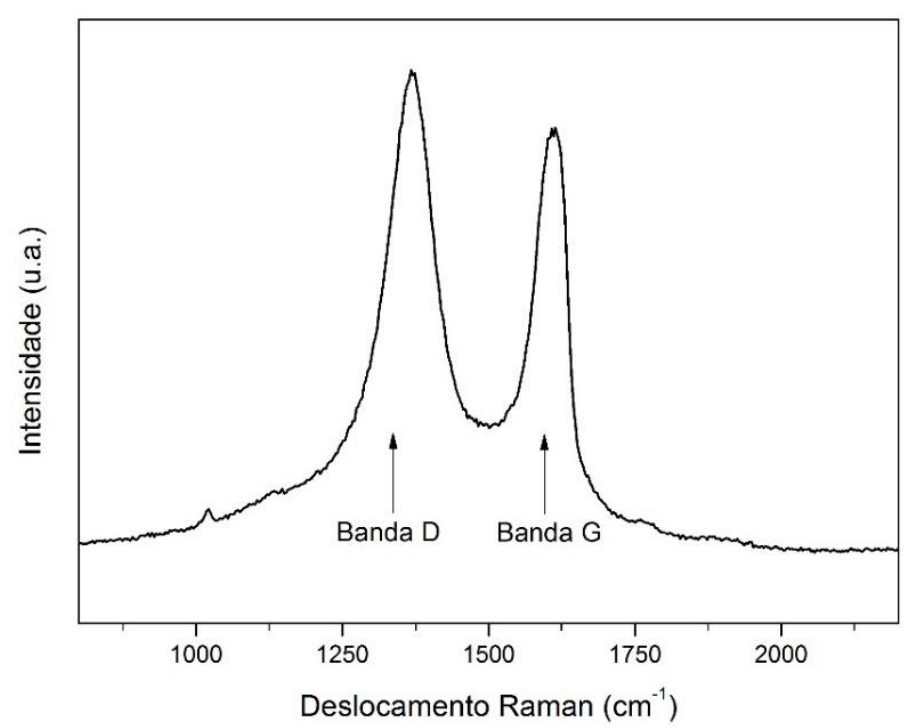

Figura 1. Espectros Raman da dispersão de GO produzida.

O padrão de difração obtido para a amostra de GO é mostrado na Figura 2. O pico identificado em $2 \theta=5,67^{\circ}$ representa a difração referente ao plano (002). Para o grafite natural, o pico referente ao mesmo plano de difração é observado em $2 \theta=$ $26,5^{\circ}$ [8]. O deslocamento do valor de $2 \theta$ está relacionado ao aumento da distância interplanar devido à presença dos grupos funcionais de oxigênio que intercalam camadas de carbono.

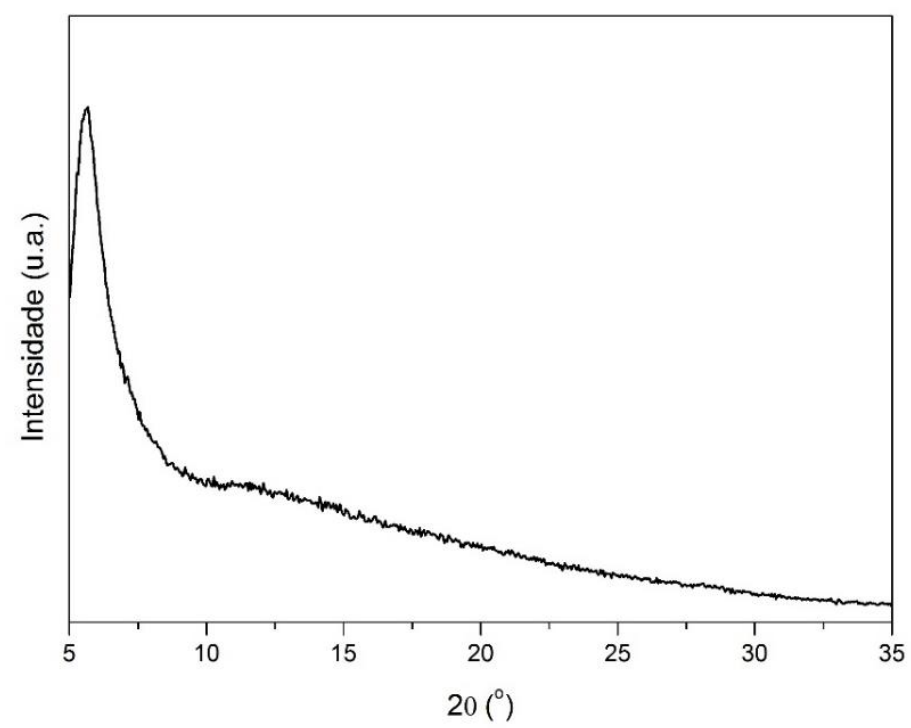

Figura 2. Difratograma da dispersão de GO produzida.

As imagens obtidas a partir dos pós de compósitos são exibidas na Figura 3. As folhas de GO estão homogeneamente distribuídas ao longo das partículas de cobre, sem pontos evidentes de aglomeração nas duas variações testadas. Com o aumento do teor de GO de $0,4 \%$ para $0,8 \%$, pode ser observada, comparando-se as imagens das Figuras 3 (a) e (b), a maior quantidade de folhas de GO distribuídas ao longo das partículas de cobre. 

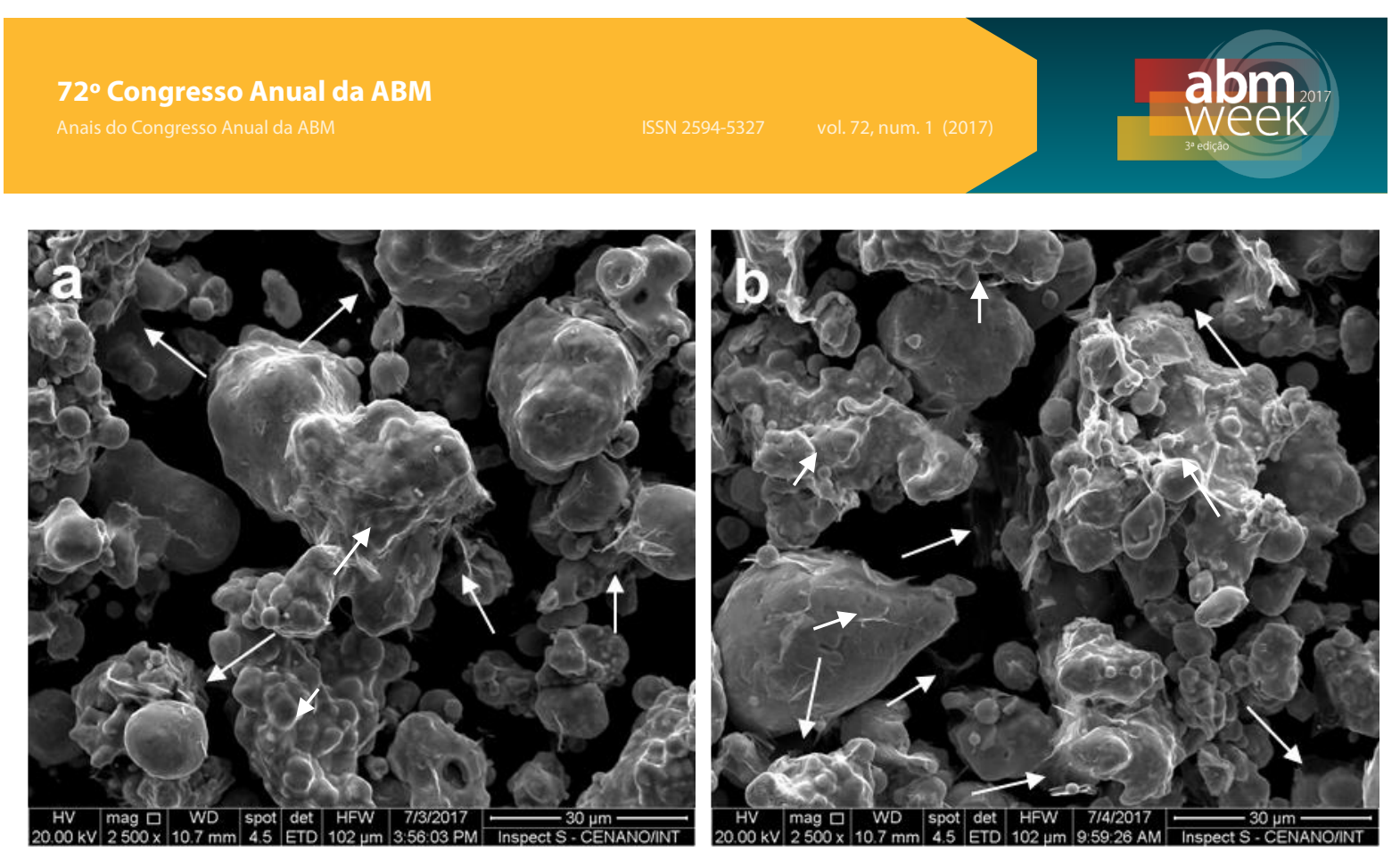

Figura 3. Imagens de MEV dos pós de compósitos (a) Cu-0,4\%GO e (b) Cu-0,8\%GO, com aumento de 2500x.

Após a sinterização, o óxido de grafeno é termicamente reduzido, os grupos funcionais são eliminados, restaurando a forma de grafeno original, passando a ser denominado óxido de grafeno reduzido. Os CP's apresentaram características muito similares entre si, diferindo-se apenas por uma pequena mudança de coloração, como mostrado na Figura 4, em função da dispersão de GO apresentar coloração marrom-escura.

(a)

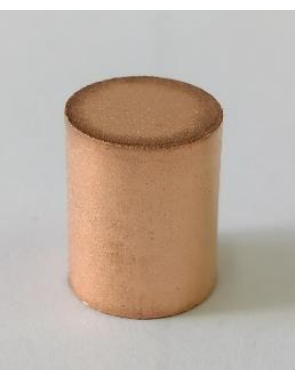

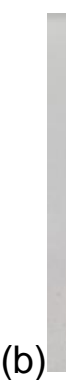

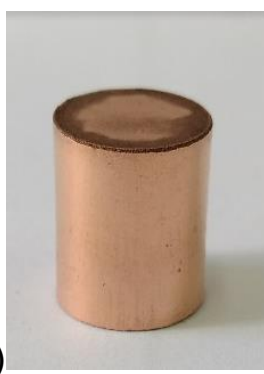

(c)

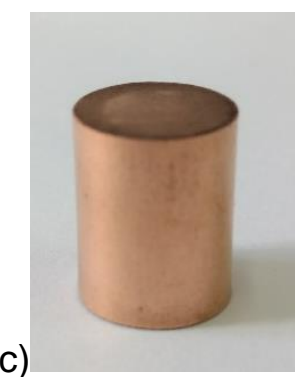

Figura 4. Fotos dos CP's de (a) Cu puro, e compósitos (b) Cu-0,4\%rGO e (c) Cu-0,8\%rGO.

Os resultados de densificação das amostras foram relacionados na Tabela 1. Pode ser notado que as densidades a verde praticamente não foram alteradas e, para todas as variações analisadas, houve um aumento da densidade após a sinterização. Com a adição de $0,4 \%$ de rGO, o aumento da densidade foi ainda mais acentuado, chegando a $94,27 \%$ de densificação. Assim, as amostras com o maior teor de grafeno $(0,4 \%)$ apresentaram valores de densidade relativamente próximas à densidade teórica do cobre puro. $O$ incremento do teor de grafeno para $0,8 \%$ causou a redução da densidade do material, em relação às demais composições avaliadas.

Tabela 1. Densificação dos corpos de prova de cobre puro e compósitos

\begin{tabular}{cccccc}
\hline Composição & $\begin{array}{c}\text { Densidade } \\
\text { a Verde } \\
\left(\mathbf{g} / \mathbf{c m}^{3}\right)\end{array}$ & $\begin{array}{c}\text { Densificação } \\
\text { a Verde } \\
(\%)\end{array}$ & $\begin{array}{c}\text { Densidade do } \\
\text { Sinterizado } \\
\left(\mathbf{g} / \mathbf{c m}^{3}\right)\end{array}$ & $\begin{array}{c}\text { Densificação } \\
\text { Sinterizado } \\
\mathbf{( \% )}\end{array}$ & $\begin{array}{c}\text { Contração } \\
\mathbf{( \% )}\end{array}$ \\
\hline Cu Puro & 7,07 & $79,12 \pm 0,24$ & 8,03 & $89,80 \pm 0,34$ & 10,68 \\
Cu-0,4\%rGo & 7,07 & $78,39 \pm 0,17$ & 8,46 & $94,27 \pm 0,15$ & 15,88
\end{tabular}


Os melhores resultados obtidos para a composição de Cu-0,4\%rGO mostraram a boa adesão entre cobre e grafeno, para pequenas adições deste reforço. Para maiores adições $(0,8 \%)$, o excesso de grafeno presente na matriz indicou a diminuição da associação entre as partículas, prejudicando o processo difusional durante a sinterização, reduzindo assim a densificação das amostras sinterizadas.

Os testes de compressão realizados nos compósitos finais deram origem às curvas tensão x deformação exibidas na Figura 5. A deformação de $40 \%$ foi usada em virtude do efeito da tensão triaxial formado na região de contato com as placas da máquina de ensaios universal. Este efeito é observado em materiais dúcteis, como o cobre, nos quais ocorre o embarrilhamento dos CP's durante o ensaio, tornando-se crítico logo após $40 \%$ de deformação, quando a geometria dos CP's possui razão $\mathrm{H}: \mathrm{D}$ próxima de $1[8]$.

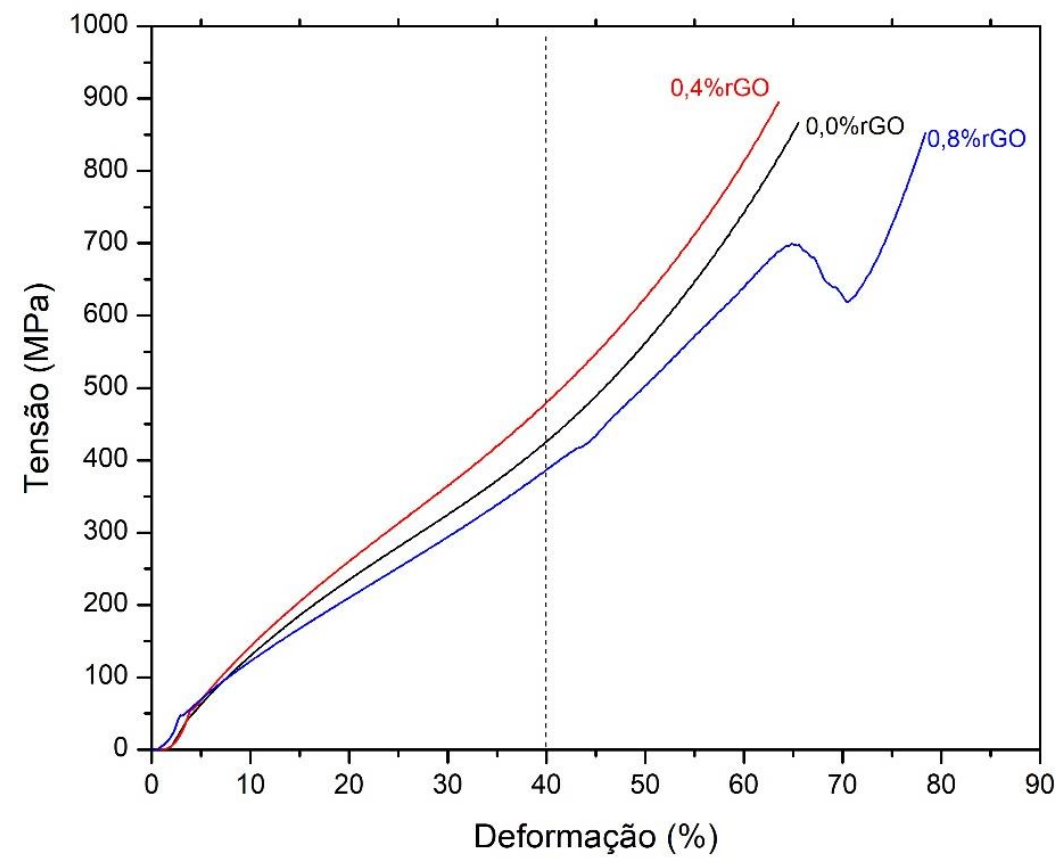

Figura 5. Curvas tensão x deformação de acordo com a composição das amostras.

A tabela 2 relaciona os resultados de resistência à compressão com $40 \%$ de deformação dos CP's. Em relação ao cobre puro, as amostras contendo $0,4 \%$ de rGO apresentaram um aumento de $14 \%$ da resistência à compressão, enquanto que para a composição de $\mathrm{Cu}-0,8 \%$ rGO houve redução em $7 \%$ da resistência.

Tabela 2. Densificação dos corpos de prova de cobre puro e compósitos

\begin{tabular}{ccc} 
Composição & $\begin{array}{c}\text { Resistência a } \\
\text { compressão } \\
\text { (40\% def.) (MPa) }\end{array}$ & $\begin{array}{c}\text { Desvio } \\
\text { Padrão } \\
\text { (MPa) }\end{array}$ \\
\hline Cu Puro & 431,76 & 4,32 \\
Cu-0,4\%rGO & 491,74 & 6,03 \\
Cu-0,8\%rGO & 403,22 & 11,41 \\
\hline
\end{tabular}

Os resultados de resistência encontrados, acompanham tendência obtida para os valores de densificação obtidos. A transferência de carga entre a matriz e o reforço 
depende fortemente da adesão superficial entre eles, o que indica que os compósitos que apresentaram maior adesão foram os com concentração de $0,4 \%$ de rGO, o que mostra que o grafeno possui, de fato, um grande potencial ao ser utilizado como reforço em compósitos com matriz de cobre.

O grafeno disperso, geralmente, adere-se envolvendo as partículas de cobre; logo, o processo difusional do cobre pode ser prejudicado, assim como constatado a partir dos resultados de densificação. Portanto, trincas serão formadas preferencialmente nas interfaces e irão se propagar ao longo da matriz de cobre, e o aumento do teor de grafeno aumenta a possibilidade de fragilização do compósito, efeito notado para a composição contendo $0,8 \%$ de rGO. Outro fator a ser considerado é a presença não só de grafeno em monocamadas, como também multicamadas, sendo possível o surgimento de trincas entre as camadas de rGO. Por conseguinte, a adição de grafeno se mostra benéfica até certo ponto, e qualquer incremento, a partir deste ponto pode ser deletério ao compósito.

Outro ponto a ser considerado é que o excesso de grafeno pode ser também um problema em virtude dos pontos de aglomeração. Regiões podem ser fragilizadas em virtude da formação de aglomerados de rGO, levando ao surgimento e propagação de trincas.

\section{CONCLUSÃO}

A espectroscopia Raman permitiu identificar as bandas D e $G$ do óxido de grafeno produzido. Por meio da difração de raios $X$ foi identificado o pico referente ao plano de difração (002), com aumento da distância interplanar, resultante da oxidação e esfoliação do grafite natural.

As análises de MEV mostraram as folhas de óxido de grafeno homogeneamente distribuídas ao longo das partículas de cobre, sem pontos de aglomeração. Foi possível identificar o aumento do teor de grafeno através das imagens obtidas.

Os resultados de resistência a compressão seguiram a tendência obtida na densificação dos CP's, onde o maior valor de resistência a compressão, com 40\% de deformação, foi referente aos compósitos com adição de $0,4 \% p$ de rGO. A adição de $0,8 \%$ p de rGO resultou na redução da resistência a compressão, em função da fragilização do material causada pelo excesso de grafeno na matriz.

\section{Agradecimentos}

Os autores agradecem a Nacional de Grafite por fornecer o grafite Graflake 99550 e à Coordenação de Aperfeiçoamento de Pessoal de Nível Superior (CAPES) e à Fundação de Amparo à Pesquisa do Estado do Rio de Janeiro (FAPERJ), pelo apoio financeiro.

\section{REFERÊNCIAS}

1 CHIAVERINI, V. Definições - Apanhado Histórico. In: Metalurgia do pó: técnicas e produtos. 4를 ed. São Paulo: Associação Brasileira de Metalurgia e Materiais, 2001.

2 NOVOSELOV, K. S.; et al. Electric Field Effect in Atomically Thin Carbon Films.

Science, v. 306, p. 666-669, 2004. 
3 GEIM, A. K.; NOVOSELOV, K. S. The rise of graphene. Nature Materials, v. 6, p. 183-191, 2007.

4 NOVOSELOV, K. S.; et al. A roadmap for graphene. Nature, v. 490, oํ 7419, p. 192-200, 2012.

$5 \mathrm{KIM}, \mathrm{Y}$; et al. Strengthening effect of single-atomic-layer graphene in metalgraphene nanolayered composites. Nature Communications, v. 4, ㄲo 2114, 2013.

6 ROURKE, J. P. et al. The Real Graphene Oxide Revealed - Stripping the Oxidative Debris from the Graphene like Sheets. Angew. Chem. Int. Ed., v. 50, p. $3173-3177,2011$.

7 ABOUELSAYED, A.; et al. Preparation, characterization, Raman, and terahertz spectroscopy study on carbon nanotubes, graphene nano-sheets, and onion like carbon materials. Materials Chemistry and Physics, v. 189, o. 127-135, 2017.

$8 \mathrm{GAO}, \mathrm{X}$; ; et al. Mechanical properties and thermal conductivity of graphene reinforced copper matrix composites. Powder Technology, v. 301, p. 601-607, 2016.

9 MESCALL, J.; PAPIRNO, R.; MCLAUGHLIN, J. Stress and Deformation States Associated with Upset Tests in Metals. Compression Testing of Homogeneous Materials and Composites, ASTM STP SOS. Richard Chait and Ralph Papirno. Eds. American Society for Testing and Materials, 1983. 